\title{
Are microbes getting up your nose?
}

\author{
Microbial aerosols in general dental practice by A. M. Bennett, M. R. Fulford, J. T. Walker, D. J. Bradshaw, \\ M. V. Martin and P. D. Marsh Br Dent J 2000; 189: 664-667
}

\section{Objective}

To measure the concentration of microbial aerosols in general dental practices and to use this information to carry out quantitative microbiological risk assessments.

\section{Methodology}

Microbial air sampling was carried out continuously during 12 treatment sessions in 6 general dental practices in the South West of England.

\section{Results}

The microbial aerosol concentration in treatment rooms was generally less than $10^{3}$ colony forming units per cubic metre of air $\left(\mathrm{cfu} \cdot \mathrm{m}^{-3}\right)$. However, in 6 out of the 12 visits, at least one peak concentration with much higher numbers of bacteria was detected. The peak concentrations were associated with increased recoveries of presumptive oral streptococci suggesting these aerosols originated from the mouths of patients. These aerosol peaks dissipated within 30 minutes and no dissemination into waiting areas was detected. The peak concentrations were associated with mechanical scaling procedures $(47 \%$ of procedures giving rise to a peak) and to a lesser extent by cavity preparation (11\%). No aerosolised blood was detected.

\section{Conclusions}

The data have been used to generate a framework for quantifying risk of exposure of staff to aerosolised microbial pathogens in general dental practice. For example, dentists and their assistants may have a slightly higher risk of exposure to Mycobacterium tuberculosis than the general public. The use of face seal masks that have been shown to protect against aerosolised microorganisms may reduce this exposure.
In Brief
- Microbiological air sampling was conducted continuously during typical treatment sessions in general dental practices (GDPs).
- At the start of treatment sessions, the numbers of aerosolised bacteria were low and typical of populated small rooms.
- Transient peaks of aerosolised micro-organisms were detected in half of the surgeries; these peaks were most often associated with scaling. No evidence of aerosol dissemination into waiting rooms was found.
- The data on aerosolised oral streptococci can be applied to risk assessment models to quantify the risk of exposure to staff and patients to aerosolised microbial pathogens. Such aerosols could be generated during treatment of, for example, asymptomatic carriers of a range of pathogens.
- The risk assessment provided no evidence of increased risk to several viral pathogens, but a potential small increased risk to exposure to Mycobacterium tuberculosis. This risk could be reduced by the correct use of effective face masks.

\section{Comment}

We know that dentists have a higher prevalence of air-borne upper respiratory tract infections but little scientific data on aerosol generation. The term aerobiology has been coined to describe the science of the aerial transport of micro-organism and other microscopic biological material in air, their deposition and ensuing consequences. This emerging science has been applied by Allan Bennett to study the common problem of aerosols in dentistry. Aerosol particles come in many shapes and sizes - large ones $(>15 \mu \mathrm{m})$ tend not to spread very far and settle out quite quickly onto horizontal (surgery worktop) surfaces. Smaller particles $(5 \mu \mathrm{m})$ have potential for a greater degree of spread (but not into the waiting rooms!). It is critical when investigating aerosol generation that all these appropriate factors are taken into account when collecting specimens. The team investigated aerosol generation during operative dentistry using three different sampling techniques to give us a comprehensive picture of the aerosols generated. Although the results may not be too surprising to those who have to clean their protective specta- cles at the end of a busy morning, it is the first study to provide an evidence-based risk assessment of aerosols in general dental practice. In particular, this paper highlights the role of universal precautions fundamental to infection control in dentistry.

Peak aerosol concentrations, which included oral micro-organisms occurred particularly with mechanical scaling procedures. Although the authors state that high volume aspiration was routinely used during the monitored procedures, it would have been interesting to have monitored the efficiency of the suction system, particularly during peak aerosol generation times. Efficient high volume aspiration is essential to reduce levels of aerosolised material during treatment. The authors also noted that some study participants were not wearing protective clothing, such as, spectacles or masks to prevent splatter from aerosols. Reassuringly no aerosolised blood was detected.

In addition to infection control issues this paper highlights the potential risks to occupational health of the dental team. Many practitioners and their team work long and hard in the same surgery environment for many years and yet little is done to provide an occupational health service. This work provides a risk assessment from aerosolised material in the dental surgery. Fortunately, most dentists in the UK are immunised against $\mathrm{TB}$ and hepatitis B and with the use of high volume aspiration to reduce aerosol generation, the risk of infection is negligible. However, there is no room for complacency and the authors raise the issue of elevated antibody levels to Legionella spp, possibly originating from dental unit water lines. Do dental staff suffer from sub-clinical Legionella infections?

This study provides excellent scientific data on aerosol generation in general dental practice. There remains a need for further studies of the role of aerosols in dental practice and its effect on the occupational health of general dental practitioners and their staff.

\section{Andy Smith}

Lecturer/Honorary Registrar in Microbiology, Infection Research Group, Glasgow Dental Hospital and School, University of Glasgow 